\title{
ESTIMATIVA DA CARGA PARASITÁRIA (Eurytrema SP) DE UM BOVINO EM FUNÇÃO DO NÚMERO DE OVOS POR GRAMA DE FEZES (OPG)*
}

\author{
ESTIMATION OF PARASITE LOAD AS A FUNCTION OF NUMBER OF EGGS PER \\ GRAM OF FAECES (EPG) IN Eurytrema SP INFECTED CATTLE
}

Pacifico Antônio Díniz BELE.M'; Mauro Rodrigues de OLIVEIRA²; Carlos Roberto PADOVANP

\begin{abstract}
RESUMO
Foram utilizados os dados de 39 bovinos oriundos de cidades da mesorregiāo do Sudoeste Paulista referentes à carga parasitária (CP) c correspondente número de ovos de Eurytrema sp por grama de fczes (OPG) para sc pesquisar, por meio de cstudos de regressão, um modelo matemático capaz de estimar a primcira a partir da segunda variável. Não obstante o fato da CP c OPG relacionarem-sc gracilmente $\left(\mathrm{R}^{2}=38,2 \%\right)$, verificou-sc que o objetivo referido pode ser alcançado cmpregando-se a seguinte relaçð̃o matemática: $\mathrm{CP}=63,1050(\mathrm{OPG}+1)^{0,5616}$. Nestas condiçð̌es, pode-se prever que os casos falsos negativos $(\mathrm{OPG}=0)$ devem albergar, nos pâncreas, uma média dc 63,1 parasitos cos casos positivos (OPG $\geq 1$ ) média igual ou supcrior a 93,2 parasitos.
\end{abstract}

UNITERMOS: Eurytrema; Carga parasitária; Bovinos

\section{INTRODUÇĀO}

Não obstantc as limitaçðcs do cmprego do OPG para cstimar cargas parasitárias, autores de renome como UENO; GONCALVES ${ }^{10}$ (1988) recomendam-no para avaliar o grau de infecçăo por ncmatóidcs gastrintestinais c Fasciola hepatica $\mathrm{cm}$ bovinos e ovinos. No quc lange ao Eurytrema sp, contudo, a literatura compulsada carece de publicaçð̋es congêneres. Este trabalho objctiva, pois, relatar os cstudos acerca da avaliaçāo de diversos modclos matemáticos para atender a cstimativa aqui proposta.

\section{MATERIAL E MÉTODO}

O material desta pesquisa foi obtido de 483 bovinos de ambos os sexos, pertencentes a diferentes raças, oriundos de diferentes propricdades rurais c que foram abatidos $\mathrm{cm}$ São Manoel, Estado de Sāo Paulo, entre 23/11/87 e 25/1/88. Utilizou-se tamberm matcrial obtido de outros dois bovinos, necropsiados na Faculdade de Mcdicina Veterinária c Zootconia da UNESP/Botucatu (FMVZ). Todos os animais eram adultos, criados $\mathrm{cm}$ regime de pasto $\mathrm{c}$ procedentes de municípios da mesorregião do Sudocstc Paulista.

Após a evisccraçăo do animal, cada pâncrcas cra cxaminado e, quando parasitado, o órgão c uma amostra mínima de $10 \mathrm{~g}$ de fezes contida na ampola retal cram recolhidos, acondicionados em sacos plásticos individuais previamente identificados c, em scguida, transportados para a FMVZ, onde se processava a contagem dos parasitos albergados $\mathrm{c}$ o número de ovos por grama de fezes (BELÉM¹, 1991).

Empregando-se estudos de análise de regressăo ${ }^{4}$, foi pesquisado um modelo matemático para estimar o número de espécimes de Eurytrema sp cxistentes no pâncreas a partir do valor do OPG.

\section{RESULTADOS E DISCUSSĀO}

Sabendo-sc quc o OPG, nos casos de infeccão por Eurytrema $\mathrm{sp}$, apresenta oscilaçōes diárias ${ }^{2.7 .8}$ este estudo, para ser conduzido $\mathrm{cm}$ melhores condiçð̄es, deveria computar diversas mcdidas de cada hospedciro c, ao final, sacrificá-los para contagem do número de parasilas. Ademais, diversos fatores poderiam ser estudados (idade, sexo, época do ano, ctc) a fim de procurar, ao máximo, cquacionar a interferência de cada um sobre o OPG c, desta forma, tentar previsões melhores sobre o grau de infecção. Todavia, a cxecução disto, na prática, sofre uma séric de limitaçðcs c envolve o acompanhamento dos animais desde o diagnóstico até o abatc. Assim, como ponto de partida, optou-sc por trabalhar com animais de matadouro que, comprovadamente, fossem parasitados por Eurytrema sp c dos quais pudesse scr obtida, com certeza, uma medida do OPG. Como se trabalharia com animais alcatoriamente cscolhidos, acreditou-se que os efcitos das flutuaçōes pudessem ser minimizados.

Ao se considerar toda a argumentação de ROCHA (1966) sobre a inadequação do OPG para estimar o grau de infeç̧ão por helmintos $\mathrm{cm}$ hospedciros, alem dos diversos fatores que podem interferir na interpretação do OPG (HUNTER; QUENOUILLE ${ }^{3}$, 1952; SOULSBY ${ }^{9}, 1982$ c UENO; GONÇALVES $\left.{ }^{10}, 1988\right)$, bem como a tese de ROCHA ${ }^{6}, 1979$ que afirma que o OPG se prestaria mais como índice epidemiológico de contaminação ambiental do que como medida da carga parasitária, podcr-sc-ia abandonar o estudo proposto antes mesmo de começá-lo. Contudo, apareccria a scguinte indagação: qual outro parâmetro, cntão, poderia scr utilizado para, ainda que grossciramente, avaliar graus de infecção antes que sintomas e prejuizos se tornassem evidentes? Na realidade, não há outro. Aliás, há de se registrar que, não obstantc as limitaçōes do cmprego do OPG para os fins mencionados, autores de renome como UENO; GONÇAL-

\footnotetext{
- Parte da tese BELEM, P.A.D. Aspectos Jigados ao diagnóstico da infecçăo por Eurytrema sp em bovinos. Botucatu, 1991 . Tese

(Doutorado) - Faculdade de Mcdicina Veterinána e \%ootecnia, Universidade Estadual Paulista - Campus de Botucatu.

1-Professor Adjunto - Universidade Federal de Viposa

2-Professor Adjunto - Faculdade de Medicina Vetcrinánia c Zootecnia da UNESP, Campus de Botucatu

3-Professor Adjunto - Instituto de Biociência da UNESP Campus de Botucatu
} 
BELfiM, P.A.D.: OIIVEIRA, M.R.; PADOVANI, C.R. Estimativa da carga parasilária (Eurytrema sp) de um bovino em função do número de ovos por grama de fezes (OPG). Braz. J. vet. Res. anim. Sci., São Paulo, v.29, p. 363-365, 1992. Suplemento.

VES $^{10}(1988)$ rocomendam-no para avaliar o grau de infecção por nematóides gastrintestinais e pela Fasciola hepatica $\mathrm{cm}$ bovinos c ovinos. No que tange ao Eurytrema sp, contudo, a literatura compulsada carece de publicaçðes congêneres.

Diante das ponderaçðes cfetuadas acima e sabendo-se que os dados foram colhidos de hospedciros infectados $\mathrm{cm}$ condiçðes naturais (c, portanto, sujcitos a cfeitos como rcinfestą̧ðes $c$ adversidades de meio que possam interferir na quantidade de fezes climinada $c$ assim fazer variar o OPG) esperava-se que, năo obstantc os cuidados tomados para execuçăo da pesquisa, estimativas da carga parasitária de indivíduos, com base no OPG, deveriam cstar sujcitas a uma margem de crro considerável. Isto, $\mathrm{cm}$ contrapartida, scria atcnuado pela premissa dc que, regra geral, é esperado, para qualquer helminto, uma rclaçðo entre quantidade de ovos nas fezcs c carga parasilária. De fato, $\mathrm{cm}$ se tratando do parasita $\mathrm{cm}$ apreço, o cstudo aqui conduzido bem confirma estas expectativas (Tab. 1) pois apenas o modclo (4) não foi significativo cstatisticamente c, dentre aqueles que o foram, a maior magnitude do $\mathrm{R}^{2}$ aproximou-se dc $40 \%$. Ou scja, a rclação acima refcrida não exclui o referido trematóide, mas as quantificaçőes da carga parasitária, com base no OPG, estăo sujcitas a crro. No entanto, há de ser lembrado, mais uma ve\%, que o OPG, a despeito de ser um índice grossciro, permancce o único capaz de dar uma idéia ao clínico do número de cspécimes de helmintos de um hospedciro sem se recorrer à necrópsia. Esta, no entanto, a despcito da incxistência de outro recurso para se conhecer a carga parasitária dos animais acometidos, c, obviamente, um procedimento inviável na rotina do clínico.

Por fim, resta verificar, dentre as várias equaçðes de regressão (Tab. 1), aquela que combine valor mais clevado do $R^{2} c$ praticidade de uso. Feita esta análise, a cscolha recai sobre a $n^{\circ} 5$, porquanto, apesar de apresentar o $R^{2}$ ligciramente mais baixo que os modelos de regressão múltipla, é muito menos complexa c pode ser transformada algebricamente para uma equação mais simples ainda:

$$
Y=63,1050(x+1)^{0.5616}
$$

O modelo matcmático acima permitc, também, inferir o número de espécimes de Eurytrema sp de um bovino quando se emprega a técnica adotada. Assim, substituindo-sc X por 1 (um), verifica-se que os casos detectados pelos exames coproparasitológicos deverão albergar média igual ou superior a 93,2 parasitos $\mathrm{c}$ os casos falsos negativos (OPG $=0$ ), em média, 63,1. Para mclhor visualizaçăo do que foi discutido, a Fig. 1 representa um diagrama de dispersão do OPG c do número de parasitas referente aos animais aqui utilizados como matcrial, além do traçado de curva resultante do modelo matcmático cscolhido para as inferĉncias citadas.

\section{CONCLUSŌES}

13) as variávcis OPG $(\mathrm{X})$ c númcro de parasitas (Eurytrema sp) dc um bovino $(Y)$ relacionam-sc gracilmentc $\left(R^{2}=\right.$ $38,2 \%)$. Nestas condiçðcs, para a obıcnção de estimativas da segunda com base na primcira, pode-se empregar o seguinte modelo matemático:

$$
\mathrm{Y}=63,1050(\mathrm{x}+1)^{0.5616}
$$

$\left.2^{\prime}\right)$ prevê-sc que casos falsos negativos ( $\left.\mathrm{OPG}=0\right)$, segundo a tócnica de cxame parasitológico empregada, alberguem, nos pâncreas, uma média de 63,1 parasitas c os casos positivos (OPG $\geq 1$ ) média igual ou supcrior a 93,2.
TABELA

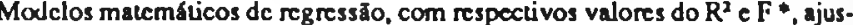
Lados para estimar o número de espécimes de Eurytrema sp (Y) de bovinos da

\begin{tabular}{|c|c|c|}
\hline Modelos & $R^{2}(\%)$ & $\mathbf{F}$ \\
\hline 1. $Y=220,7718+7,5275(x+1)$ & 21,5 & 10,12 \\
\hline 2. $Y=454,9769-\frac{420.2416}{x+1}$ & 18.7 & 8.49 \\
\hline 3. $Y=66,0557+78,9224(x+1)^{12}$ & 28.2 & 14.56 \\
\hline 4. $Y=306,9758+0,0688(x+1)^{2}$ & 9,2 & 3.77 \\
\hline 5. $\ln Y=4,1448+0,5616 \ln (x+1)$ & 38,2 & 22,84 \\
\hline 6. $Y=60,1217+132,1792 \ln (x+1)$ & 29,1 & 15,21 \\
\hline 7. $\ln Y=5,9257-\frac{2.2134}{x+1}$ & 37,6 & 22,26 \\
\hline 8. $\ln Y=4,9173+0,0269(x+1)$ & 19.9 & 9.19 \\
\hline 9. $\sqrt{Y}=13,4394+0,2015(x+1)$ & 23,0 & 11,06 \\
\hline 10. $\sqrt{Y}=20,1734-\frac{13.1779}{x+1}$ & 27.4 & 13,06 \\
\hline 11. $\sqrt{Y}=9,1157+2,1628(x+1)^{16}$ & 31,7 & 17.16 \\
\hline 12. $\sqrt{Y}=15,7412+0,0019(x+1)^{2}$ & 10,0 & 4,10 \\
\hline 13. $\ln \sqrt{Y}=2,0716+0,2809 \ln (x+1)$ & 38,1 & 22,79 \\
\hline 14. $\sqrt{Y}=8,6059+3,7788 \ln (x+1)$ & 35.6 & 20.43 \\
\hline 15. $\ln \sqrt{Y}=2,9622-\frac{1,1063}{x+1}$ & 37,5 & 22,22 \\
\hline 16. $\operatorname{In} \sqrt{Y}=2,4578+0,0235(x+1)$ & 19.9 & 9,21 \\
\hline 17. $Y=102,0230+25,9255 x-0,2800 x^{2}$ & 38,0 & 11,04 \\
\hline 18. $\begin{aligned} Y= & 150,2029+11,8630 x+0,3153 x^{2}- \\
& -0,0057 x^{3}\end{aligned}$ & 41,0 & 8,11 \\
\hline 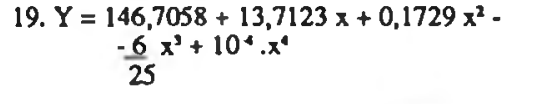 & 41,0 & 5.91 \\
\hline 20. $\sqrt{Y}=9,6017+0,7034(x+1)-\frac{37}{50}(x+1)^{2}$ & 40,3 & 12.17 \\
\hline \multicolumn{3}{|l|}{ 21. $\sqrt{Y}=10,4715+0,4921(x+1)+\frac{4}{25}(x+1)^{2}+$} \\
\hline$+\frac{x+1}{10000}$ & 41,2 & 8,17 \\
\hline 22. $\begin{aligned} \sqrt{Y}= & 9.555+0.841(x+1) \\
& +\frac{(x+1)^{3}}{10000}-\frac{(x+1)^{4}}{10000}\end{aligned}$ & 42,0 & 6,16 \\
\hline
\end{tabular}
mesortegiäo do Sudocsic Paulista cm funç̧̃ do OPG $(X)$

\section{SUMMARY}

The parasite load (P) and correspondent number of eggs per gram of facces (EPG) were counted in 39 Eurytrema sp infected cattle from Southwestem localitics of São Paulo Statc. By statistical studics involving regression, the following mathematic model was obtained: $P=63,1050(E P G+1)$ $0.5616 ; R^{2}=38.2 \%$. In such conditions, false negative cases $(E P G=0)$ should harbour an average of 63,1 flukes in its pancreas and positive ones (EPG $\geq 1$ ) an average equivalent or superior to 93,2 .

UNITERMOS: Eurytrema; Parasit load; Catle

\section{REFERÊNCIAS BIBLIOGRÁFICAS}

01-BELÉ, P. A. D. Aspectos ligados ao diagnóstico da infeç̧ão por Eurytrema spem bovinos. Botucatu, 1991. Tese (Douto- 
rado) - Faculdade de Medicina Veterinária e Zootecnica. Universidade Estadual Paulista, Campus de Botucatu.

02-CHINONE, S.; ITAGAKI, H. Development of Eurytrema pancreatlcum (TREMATODA). II. Development in definitive hosts. Bull. Azabu vet. Coll., v.1, p. 73-81, 1976.

03-HUNTER, G. C.; QUENOUILLE, M.H. Statistical examination of the worm egg count sampling tochnique for sheep. J. Helminth., v. 26, p. 157-70, 1952.

04-OSTLE, B. Estadistica aplicada. 3.ed, Mexico, Limusa - Wiley, 1973.

05-ROCHA, C. A. O sentido da contagem de ovos de helmintos por grama de fezes do hospedeiro. R. Med. Vet., v.2, p.63-4, 1966.

06-ROCHA, U.F. Quantificações e estabclecimento de parâmetros e modelos matemáticos para estudo das interações do tipo hospodciro-parasito. In: SEMINÁRIO NACIONAL SOBRE PARASITOSES DOS BOVINOS, 1., Campo Grande, 1979. Anais. Campo Grande, EMBRAPA, 1979. p.47-58.
07-SAKAMOTO, T.; KONO, I.; MOHRI, S. Studies on Eurytrema collomaticum. IV. Comparison between anthelmintic effects of drugs against Eurytrema coelomaticum "in vitso" and "in vivo". J. Fac. Agric. Iwate Unlv., v. 17, p.211-27, 1984.

08-SAKAMOTO, T.; KONO, I.; YASUDA, N.; YAMAMOTO, Y.; NAKAGAWA, H. Studies on Eurytrema coelomaticum. II. The anthelmintic efficiency of nitroxynil and praziquantel against Eurytrema coelomaticum in cattle. Mem. Fac. Agric. Kagoshima Univ., v. 16, p. 93-101, 1980.

09-SOULSBY . E. J. L. Helminths, artropodos and protozoa of domesticated animals. 7. ed. Philadelphia, Lea \& Febiger, 1982.

10-UENO, H.; GONÇALVES, P. C. Manual para diagnóstico das helmintoses de ruminantes. 2. ed. T6́quio, Japan International Cooperation Agency, 1988.

Recebido para publicaçảo cm 15/05/1992 Aprovado para publicação cm 10/09/1992

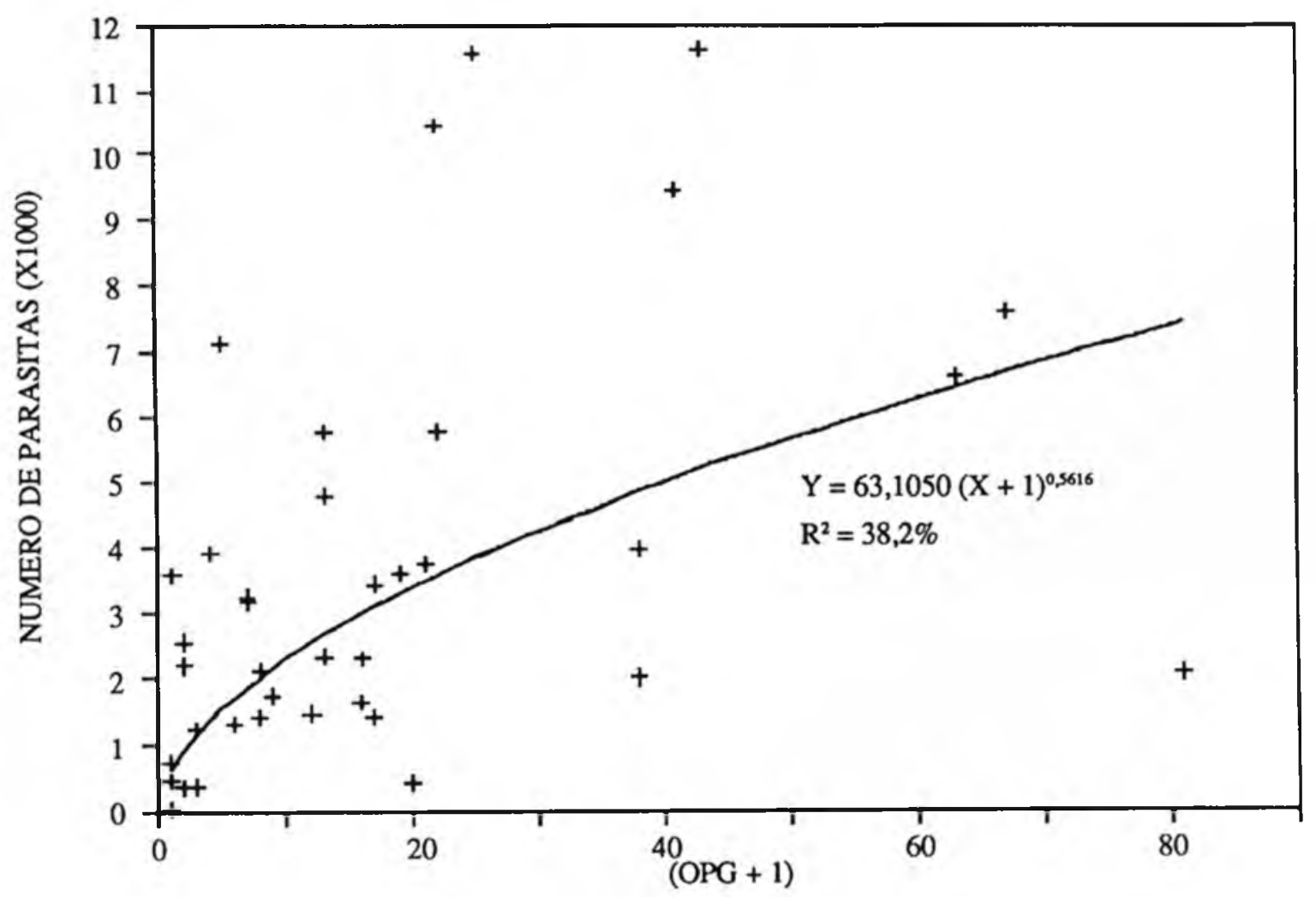

Iigura 1

Equaçăo da regressão e diagrama de dispersão do OPG e número de espécimes de Eurytrema sp de 38 bovinos abatidos cm São Manocl, São Paulo, entre 23/11/87 e 25/1/88, e de um outro necropsiado na FMVZUNESP - Botucatu, STo Paulo, em 8/12/87, todos infectados nauralmente e oriundos de municípios da mesorregiăo do Sudocste Paulista 